\title{
Lithium abundance in the metal-poor open cluster NGC $2243^{\star, \star \star}$
}

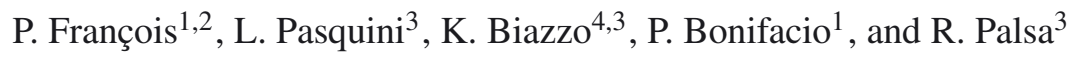 \\ 1 GEPI - Observatoire de Paris, 64 avenue de l'Observatoire, 75014 Paris, France \\ e-mail: patrick. francois@obspm.fr \\ 2 UPJV - Université de Picardie Jules Verne, 80000 Amiens, France \\ 3 ESO - European Southern Observatory, Karl-Schwarzschild-Str. 3, 85748 Garching bei München, Germany \\ 4 INAF - Capodimonte Astronomical Observatory, via Moiariello 16, 80131 Naples, Italy
}

Received 19 December 2012 / Accepted 20 February 2013

\begin{abstract}
Context. Lithium is a fundamental element for studying the mixing mechanisms acting in the stellar interiors, for understanding the chemical evolution of the Galaxy and the Big Bang nucleosynthesis. The study of Li in stars of open clusters (OC) allows a detailed comparison with stellar evolutionary models and permits us to trace its galactic evolution. The OC NGC 2243 is particularly interesting because of its low metallicity $([\mathrm{Fe} / \mathrm{H}]=-0.54 \pm 0.10 \mathrm{dex})$.

Aims. We measure the iron and lithium abundance in stars of the metal-poor OC NGC 2243. The first aim is to determine whether the Li dip extends to such low metallicities, the second is to compare the results of our Li analysis in this OC with those present in 47 Tuc, a globular cluster of similar metallicity.

Methods. We performed a detailed analysis of high-resolution spectra obtained with the multi-object facility FLAMES at the ESO VLT $8.2 \mathrm{~m}$ telescope. Lithium abundance was derived through line equivalent widths and the OSMARCS atmosphere models. Iron abundances from Fe I and Fe II lines have also been measured and used to check the atmospheric model parameters.

Results. The Li line is detected in 27 stars confirmed as likely cluster members by repeated radial velocity measurements. We determine a Li dip center of $1.06 M_{\odot}$, which is much smaller than that observed in solar metallicity and metal-rich clusters. This finding confirms and strengthens the conclusion that the mass of the stars in the Li dip strongly depends on stellar metallicity. The mean $\mathrm{Li}$ abundance of the cluster is $\log n(\mathrm{Li})=2.70$ dex, which is substantially higher than that observed in 47 Tuc. We estimated an iron abundance of $[\mathrm{Fe} / \mathrm{H}]=-0.54 \pm 0.10 \mathrm{dex}$ for NGC 2243 , which is similar (within the errors) to previous findings. The $[\alpha / \mathrm{Fe}]$ content ranges from $0.00 \pm 0.14$ for Ca to $0.20 \pm 0.22$ for Ti, which is low when compared to thick disk stars and to Pop II stars, but compatible with thin disk objects. We found a mean radial velocity of $61.9 \pm 0.8 \mathrm{~km} \mathrm{~s}^{-1}$ for the cluster.

Conclusions. We confirm a correlation between the Li dip cool-side position in mass as a function of the cluster's [Fe/H]. The Li abundance found in the metal-poor OC NGC 2243 agrees well with results obtained for the interstellar medium in the Small Magellanic Cloud having similar low metallicity. This value of $\mathrm{Li}$ is comparable to the primordial Li abundance deduced from WMAP measurements, therefore putting strong constraints on the models of Li enrichment during the early history of our Galaxy.
\end{abstract}

Key words. stars: late-type - open clusters and associations: individual: NGC 2243 - techniques: spectroscopic

\section{Introduction}

The measurement of the Li abundance of turnoff and dwarf stars in OCs of different ages and metallicities is a very powerful tool that can be used to investigate a number of topics: the possibility of sampling stars of different evolutionary status and original mass allows to understand how this element is destroyed and to test different evolutionary models and mixing mechanisms (Boesgaard \& Tripicco 1986; Pasquini 2000; Randich et al. 2000, 2003; Smiljanic et al. 2010). This is possible because for OC stars we can obtain reliable ages, metallicities, masses, and evolutionary stages from precise photometry and high-resolution spectroscopy. Cluster stars are the most powerful targets that can be studied to disentangle the effect of each of these parameters on mixing phenomena. Since $\mathrm{Li}$ is burnt through $(p, \alpha)$ reactions at a temperature of $\simeq 2.5 \times 10^{6} \mathrm{~K}$, differences in $\mathrm{Li}$ abundance in stars of different masses and temperatures can be used to

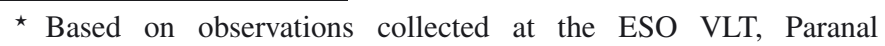
Observatory (Chile).

$\star \star$ Tables 2 and 3 are available in electronic form at http://www. aanda.org constrain the physical processes that bring the surface material to the interior of the star.

The cluster we have studied, NGC 2243, is particularly interesting because it is one of the most metal-poor OCs accessible for high-resolution spectroscopy. It is almost as metal-poor as the globular cluster 47 Tucanae, considered "metal rich" among globular clusters with a metallicity of $[\mathrm{Fe} / \mathrm{H}]=-0.76 \pm 0.04 \mathrm{dex}$ (Koch \& McWilliam 2008). The comparison of the Li abundances in the two clusters is very interesting, since, in spite of the similar metallicity, NGC 2243 is much younger than 47 Tuc. The so-called "Li dip", i.e., the disappearance of $\mathrm{Li}$ in the spectra of stars over a narrow temperature range, is a feature that is present in OCs, but in none of the globular clusters. It is interesting to investigate whether it is present also in an OC with a low metallicity comparable to that of "metal-rich" globular clusters. Another interesting characteristic of OCs that are young enough to have hot main sequence stars on the blue side of the dip (see, e.g., Sestito \& Randich 2005, and references therein) is that hot stars out of the "Li dip" show a high level of Li abundance, close to the abundance present in the interstellar medium (ISM) when they formed. This initial Li abundance in metal-poor clusters will provide precious data to be compared with models of 
Li evolution in the Galaxy (e.g., Matteucci 2010) and primordial nucleosynthesis (Cyburt et al. 2008).

The first determination of the $\mathrm{Li}$ abundance in this cluster was performed by Hill \& Pasquini (2000). They found a mean $\mathrm{Li}$ abundance of $\log n(\mathrm{Li})=2.35$ in the four stars for which they could detect $\mathrm{Li}$. These authors hinted at the presence of a Li dip, but could not confirm it, given their limited sample.

Here, we present a new study of elemental abundances in NGC 2243, based on high-resolution spectra obtained with FLAMES on the VLT. In Sect. 2 we briefly describe the cluster. The observations and data reduction are given in Sect. 3 , while the data analysis is discussed in Sect. 4. The results, discussion, and conclusions are presented in Sects. 5-7.

\section{NGC 2243: an old metal-poor open cluster}

NGC 2243 is an old and metal-poor OC located towards the anti-center at $R_{\mathrm{G}}=10.76 \mathrm{kpc}$ and $z=1.1 \mathrm{kpc}$ away from the plane of the Milky Way $\left(l_{\mathrm{II}}=239^{\circ} .478, b_{\mathrm{II}}=-18^{\circ} .014\right)$. The first CCD photometry of NGC 2243 was obtained by Bonifazi et al. (1990) who found an age of $4 \pm 1 \mathrm{Gyr}$, a metallicity of $[\mathrm{Fe} / \mathrm{H}]=$ $-0.80 \pm 0.10$, and a distance modulus $(m-M)_{0}=12.8 \pm 0.2$. From $B V$ CCD photometry, Bergbusch et al. (1991) derived a distance modulus of 13.05 (assuming $E(B-V)=0.06$ ), a metallicity of -0.47 dex, an overabundance of oxygen $[\mathrm{O} / \mathrm{Fe}]=$ +0.23 , and an age of 5 Gyr. Gratton \& Contarini (1994) confirmed this low metallicity using high-resolution spectra. They determined the iron abundance in two giants of the cluster and found $[\mathrm{Fe} / \mathrm{H}]=-0.48 \pm 0.15 \mathrm{dex}$ and $[\mathrm{Ca} / \mathrm{Fe}]=0.18 \pm 0.17 \mathrm{dex}$. Fitting the data of Bergbusch et al. (1991) to new isochrones, assuming core overshooting, Vandenberg et al. (2006) obtained $[\mathrm{Fe} / \mathrm{H}]=-0.61,[\alpha / \mathrm{Fe}]=0.3$, an age of $3.1 \mathrm{Gyr}$ and $(m-M)_{V}=$ 13.15 , assuming $E(B-V)=0.062$. From VI CCD photometry, Kaluzny et al. (1996) found $E(V-I)=0.10 \pm 0.04$. Kaluzny et al. (2006) extended their study of NGC 2243 to the analysis of detached eclipsing binaries. They derived a distance modulus $(m-M)_{V}=13.24 \pm 0.08$. Using model age-luminosity and age-radius relations for the binary system NV CMa, a member of the cluster, they obtained an age of $4.35 \pm 0.25 \mathrm{Gyr}$ assuming a metallicity $[\mathrm{Fe} / \mathrm{H}]=-0.525$. However, these values are sensitive to the adopted metallicity, a change of -0.08 dex in the metallicity leading to an age decrease of $\simeq 0.5 \mathrm{Gyr}$.

\section{Observations and data reduction}

The spectra were obtained in four hours in four observing nights during the FLAMES+GIRAFFE Science Verification program. FLAMES (Pasquini et al. 2002) is mounted at the Nasmyth A platform of the $8.2 \mathrm{~m}$ UT2/Kueyen of the VLT. Observations were carried out in MEDUSA mode with two GIRAFFE highresolution (HR) settings and with a single $2 \mathrm{~K} \times 4 \mathrm{~K}$ EEV CCD (15 $\mu$ m pixels). In particular, the employed settings were Filter N. 14 (échelle grating order N. 9) with central wavelength $651.5 \mathrm{~nm}$ and wavelength range between 638.3 and $662.6 \mathrm{~nm}$, and Filter N. 15 (échelle order N. 8) with the central wavelength $679.7 \mathrm{~nm}$ and wavelength range between 659.9 and $695.5 \mathrm{~nm}$. The resolution was $R \sim 28800$ and $R \sim 19300$, respectively. The log of the observations is given in Table 1 .

We selected from the catalog of Kaluzny et al. (1996) the main-sequence (MS), turnoff (TO), and (sub-)giant stars with 14 . $0 \leq V \leq 17.5$ and, thanks to the GIRAFFE configuration, we were able to observe 100 targets (Tables 2-4). Separate exposures were obtained to be able to identify short and intermediate
Table 1. Log of the observations.

\begin{tabular}{lccccc}
\hline \hline $\begin{array}{l}\alpha \\
\left({ }^{\circ}\right)\end{array}$ & $\begin{array}{c}\delta \\
\left({ }^{\circ}\right)\end{array}$ & $\begin{array}{c}\text { Date } \\
(\mathrm{d} / \mathrm{m} / \mathrm{y})\end{array}$ & $\begin{array}{c}\text { UT } \\
(\mathrm{h}: \mathrm{m}: \mathrm{s})\end{array}$ & $\begin{array}{c}t_{\exp } \\
(\mathrm{s})\end{array}$ & Filter \\
\hline 97.40327 & -31.28795 & $27 / 01 / 2003$ & $04: 12: 26$ & 3600 & HR15 \\
97.40331 & -31.28800 & $28 / 01 / 2003$ & $05: 49: 49$ & 3600 & HR14 \\
97.40326 & -31.28808 & $29 / 01 / 2003$ & $03: 29: 14$ & 3600 & HR15 \\
97.40333 & -31.28806 & $31 / 01 / 2003$ & $02: 14: 15$ & 3600 & HR14 \\
\hline
\end{tabular}

period binaries by comparing the radial velocities at different epochs. The spectra have a typical signal-to-noise ratio $(\mathrm{S} / \mathrm{N})$ of 30-40.

The observations were reduced using the version 1.12 of the GIRAFFE Base-Line Data Reduction Software (girBLDRS ${ }^{1}$; Blecha et al. 2000), which is a set of python scripts/modules and a $\mathrm{C}$ library. With it we removed the instrumental signature from the observed data, subtracting the bias and dividing by the normalized flat-field. Flat-field acquisitions were also used to trace the position of all the fibers, and to derive the parameters for the optimal extraction of the science exposures. Finally, the wavelength calibration was obtained using the day-time Th-Ar lamp exposure.

Radial velocities were measured using the $\mathrm{IRAF}^{2}$ package FXCOR, which cross-correlates the observed spectrum with a template. As a template, we used a solar spectrum acquired with FLAMES/GIRAFFE. Finally, the heliocentric correction was applied. We considered only the spectra acquired with the HR14 setting, as it leads to the radial velocity measurements with the lowest rms uncertainties (around $1 \mathrm{~km} \mathrm{~s}^{-1}$ ), compared to the $2.5 \mathrm{~km} \mathrm{~s}^{-1}$ obtained with the HR15 setting. The two spectra/star/setting were finally co-added to perform the spectroscopic determination of elemental abundance (and effective temperature).

The GIRAFFE solar spectra ${ }^{3}$, taken with the same setups of our observations, are used throughout this work for spectroscopic comparison with the stars and the synthetic spectra. Each solar spectrum was obtained by averaging most of the GIRAFFE spectra (some spectra show clear flat field problems and were not used) and it has a nominal $\mathrm{S} / \mathrm{N}$ ratio above 400 .

\section{Data analysis}

\subsection{Radial velocities}

The evolutionary status of the analyzed stars is indicated by their position in the color-magnitude diagram (CMD) shown in Fig. 1, in which our sample is shown with circles. The stars are mostly MS, TO, post-TO, subgiants, and giants, where the region around the TO is very well sampled.

Having several exposures for each object allows the comparison of the single radial velocity (RV) for each star. We have retained as most-probable single members all those showing RV variations smaller than $2 \mathrm{~km} \mathrm{~s}^{-1}$ in the two exposures acquired and having a mean velocity within about 3 sigma $\left(\approx 3 \mathrm{~km} \mathrm{~s}^{-1}\right)$ from the median cluster RV. The final values of the difference in the computed RV is given in Fig. 2, as well as the distribution,

\footnotetext{
1 http://girbldrs. sourceforge.net/

2 IRAF is distributed by the National Optical Astronomy Observatory, which is operated by the Association of the Universities for Research in Astronomy, Inc. (AURA) under cooperative agreement with the National Science Foundation.

3 http://www . eso.org/observing/dfo/quality/GIRAFFE/ pipeline/solar.html
} 


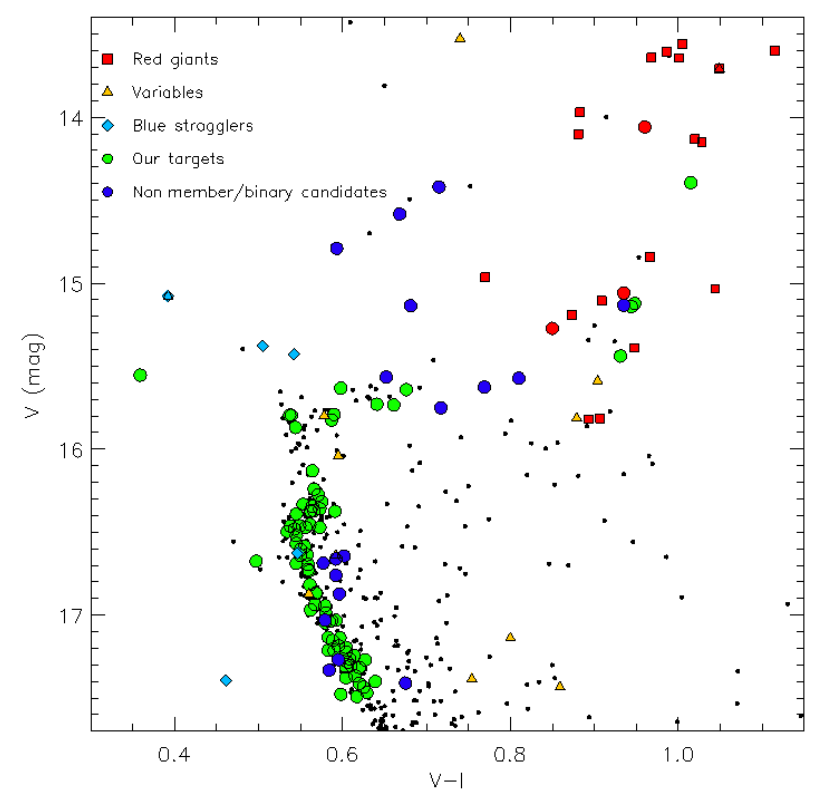

Fig. 1. Portion of the CMD of NGC 2243. Small dots represent $V$ and $V-I$ data taken from Kaluzny et al. (1996), while squares, triangles, and diamonds mark the positions of red giants, variables, and blue stragglers taken from the literature (WEBDA database). Circles refer to our targets, where the position of the most-probable single stars and binaries or non-members (from our radial velocity analysis) are marked in green and blue, respectively, while red giants from the literature (WEBDA database) are in red.

which is well represented by a Gaussian with a $\sigma$ of $0.8 \mathrm{~km} \mathrm{~s}^{-1}$. The final mean radial velocities are shown in Fig. 3 as a function of the $V-I$ color. Most of the RV are concentrated at around $62 \mathrm{~km} \mathrm{~s}^{-1}$, close to the expected radial velocity of the cluster. A total of 82 stars are retained as bona fide RV single members because their RV distribution can be well approximated by a Gaussian with central RV of $61.9 \pm 0.8 \mathrm{~km} \mathrm{~s}^{-1}$ as shown in Fig. 4. In Tables 2-4 the RV values of the stars with well-measured $\mathrm{Li}$ abundances, upper-limited $\mathrm{Li}$ abundances, and most-probable binaries or non-members are listed.

\subsection{Atmospheric parameters}

We used the photometric results for $V-I$ colors from Kaluzny et al. (1996) to derive the effective temperatures from the relations of Casagrande et al. (2010). We also attempted to measure effective temperatures from our GIRAFFE spectra, using the $\mathrm{H} \alpha$ wings, but the results were not convincing when comparing these effective temperatures with photometry. Most likely the flat field procedure adopted did not succeed in fully removing the rather strong blaze present in the spectra, possibly because of the relatively close position of the $\mathrm{H} \alpha$ line to the spectrum edge.

The surface gravities for the program stars were obtained from the photometric data quoted above and computed using the standard relation given in Eq. (1). We adopted the following solar values: $\log g_{\odot}=4.44 \mathrm{dex}, T_{\mathrm{eff}, \odot}=5790 \mathrm{~K}, M_{\mathrm{bol}, \odot}=$ $4.72 \mathrm{mag}$. The bolometric corrections were computed using the relation of Alonso et al. (1999). We assumed a TO mass of $1.15 M_{\odot}$ and a distance modulus of 13.15 . We note that even if the adopted turnoff mass was wrong by $25 \%$, the errors in computing $\log g$ would be less than 0.1 dex.

$\log g_{*}=\log g_{\odot}+\log \frac{M_{*}}{M_{\odot}}+\log \frac{T_{\mathrm{eff}, *}}{T_{\mathrm{eff}, \odot}}+0.4\left(M_{\mathrm{bol}, *}-M_{\mathrm{bol}, \odot}\right)$.

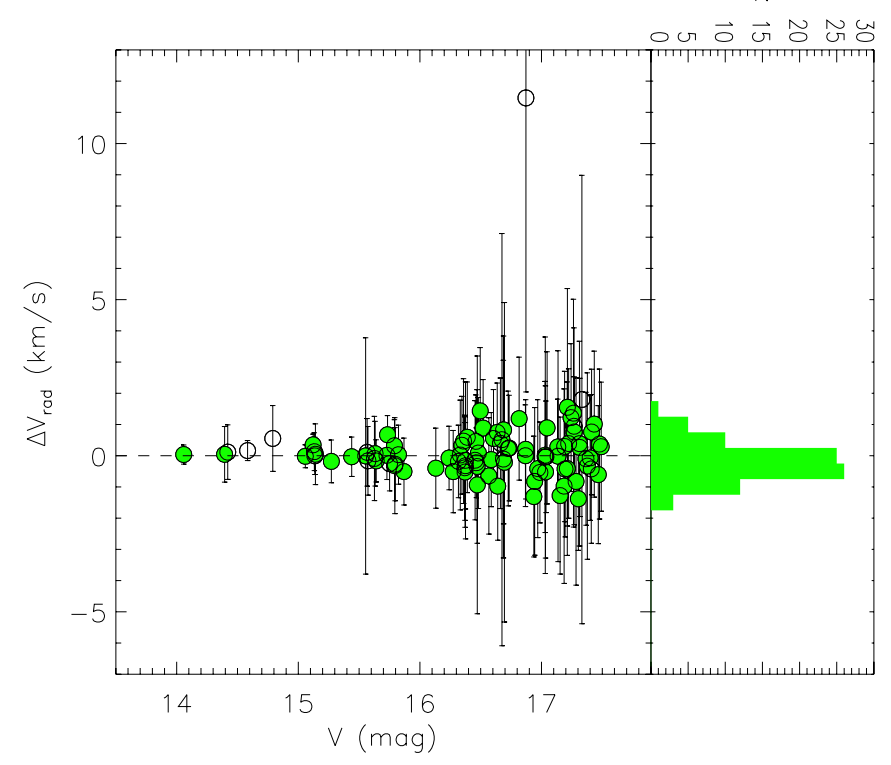

Fig. 2. Final radial velocity variations versus $V$ magnitude. The right part of the figure represents the histogram of the computed mean RV differences. Open symbols refer to the final selection of binaries or non-members.

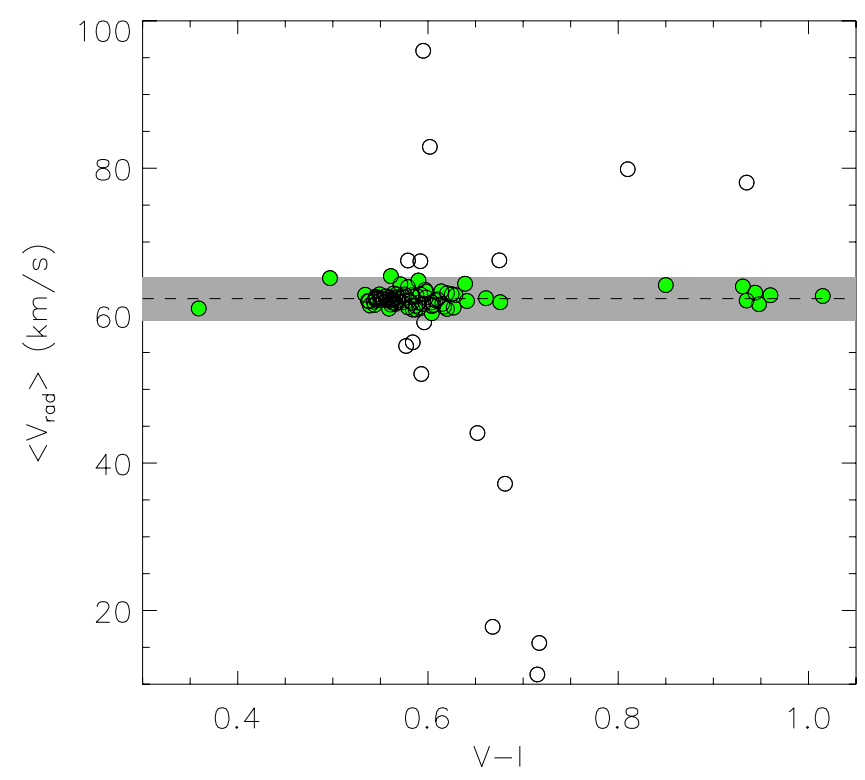

Fig. 3. Mean radial velocities as a function of the $V-I$ color. The dashed line represents the final mean $\mathrm{RV} \sim 62 \mathrm{~km} \mathrm{~s}^{-1}$, while the filled area is the $\pm 3 \sigma$ level $\left(\sim 3 \mathrm{~km} \mathrm{~s}^{-1}\right)$. Open symbols refer to the final selection of binaries or non-members.

The microturbulence velocities were computed using the relation of Edvardsson et al. (1993).

\section{Results}

\subsection{Lithium abundance}

The equivalent widths of the $\mathrm{Li}$ resonance doublet at $\lambda=$ $670.78 \mathrm{~nm}$ was measured using the code FITLINE written by François. Details on the algorithm can be found in Lemasle et al. (2007). The line was measurable in 27 stars. When the lines were not detectable, an upper limit for the equivalent width of the line 


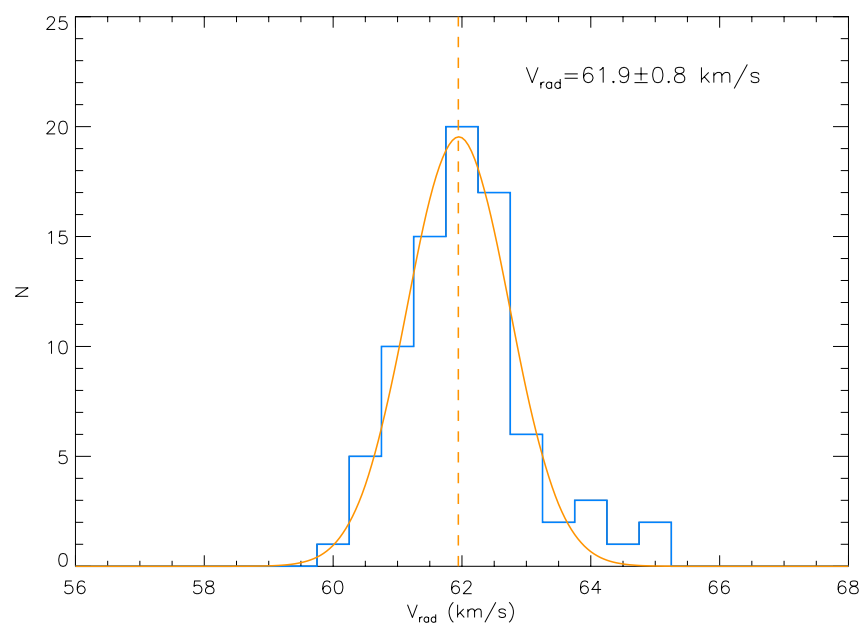

Fig. 4. Histogram of the radial velocity distribution of the 82 mostprobable single members selected in NGC 2243 (continuous line). A Gaussian fit to the distribution of member stars is also displayed, with a mean value of $\left\langle V_{\mathrm{rad}}\right\rangle=61.9 \pm 0.8 \mathrm{~km} \mathrm{~s}^{-1}$ (dashed line).

Table 4. Radial velocities of the most-probable binary stars and non-members.

\begin{tabular}{lcccc}
\hline \hline Object & $\begin{array}{c}V \\
(\mathrm{mag})\end{array}$ & $\begin{array}{c}V_{\mathrm{rad}}^{1} \\
\left(\mathrm{~km} \mathrm{~s}^{-1}\right)\end{array}$ & $\begin{array}{c}V_{\mathrm{rad}}^{2} \\
\left(\mathrm{~km} \mathrm{~s}^{-1}\right)\end{array}$ & $\begin{array}{c}\left\langle V_{\mathrm{rad}}\right\rangle \\
\left(\mathrm{km} \mathrm{s}^{-1}\right)\end{array}$ \\
\hline 343 & 14.790 & $52.4 \pm 0.7$ & $51.8 \pm 0.7$ & $52.1 \pm 0.5$ \\
92 & 15.751 & $15.5 \pm 0.7$ & $15.7 \pm 0.5$ & $15.6 \pm 0.4$ \\
671 & 16.644 & $56.4 \pm 1.2$ & $109.4 \pm 2.2$ & $82.9 \pm 1.2$ \\
182 & 15.136 & $37.2 \pm 0.4$ & $37.2 \pm 0.4$ & $37.2 \pm 0.3$ \\
153 & 14.583 & $17.9 \pm 0.2$ & $17.7 \pm 0.2$ & $17.8 \pm 0.2$ \\
495 & 17.268 & $96.4 \pm 2.3$ & $95.5 \pm 2.2$ & $95.9 \pm 1.6$ \\
1020 & 17.332 & $57.3 \pm 4.2$ & $55.5 \pm 5.8$ & $56.4 \pm 3.6$ \\
1906 & 14.420 & $11.4 \pm 0.7$ & $11.3 \pm 0.5$ & $11.3 \pm 0.4$ \\
370 & 17.409 & $67.3 \pm 1.5$ & $67.7 \pm 1.8$ & $67.5 \pm 1.2$ \\
2003 & 16.872 & $64.8 \pm 5.0$ & $53.4 \pm 8.0$ & $59.1 \pm 4.7$ \\
2018 & 15.573 & $79.8 \pm 0.9$ & $80.0 \pm 0.6$ & $79.9 \pm 0.5$ \\
1438 & 16.662 & $67.6 \pm 1.5$ & $67.2 \pm 1.4$ & $67.4 \pm 1.0$ \\
1410 & 16.760 & $\ldots{ }^{*}$ & $\ldots{ }^{*}$ & $\ldots{ }^{*}$ \\
1631 & 16.687 & $55.8 \pm 2.8$ & $55.9 \pm 1.5$ & $55.9 \pm 1.6$ \\
794 & 15.132 & $78.1 \pm 0.2$ & $78.0 \pm 0.6$ & $78.1 \pm 0.3$ \\
1831 & 15.566 & $44.1 \pm 0.9$ & $44.0 \pm 0.6$ & $44.1 \pm 0.5$ \\
1930 & 15.626 & $-18.0 \pm 1.1$ & $-17.9 \pm 0.8$ & $-17.9 \pm 0.7$ \\
1454 & 17.030 & $67.5 \pm 1.4$ & $67.5 \pm 2.0$ & $67.5 \pm 1.2$ \\
\hline
\end{tabular}

Notes. ${ }^{(*)}$ Almost continuum-type spectra.

was established via the Cayrel formula (Cayrel 1988) and translated into an upper limit for the $\mathrm{Li}$ abundance.

We then derived $\mathrm{Li}$ abundances for the measured line equivalent widths using Turbospectrum (Alvarez \& Plez 1998) taking the most updated version of the OSMARCS atmosphere models (e.g., Gustafsson et al. 2008; Plez 2008). Plane parallel 1D models have been used for the computations. We adopted the Grevesse \& Sauval (1998) solar abundances. In Table 2 the list of stars for which the $\mathrm{Li}$ abundances could be measured is given, while Table 3 lists the estimation of the $\mathrm{Li}$ abundance upper limits for the remaining stars of the sample.

\subsubsection{Elemental abundance of other elements}

The line list used to compute the iron abundance $([\mathrm{Fe} / \mathrm{H}])$ was taken from Pace et al. (2010). We used this list to measure welldefined unblended Fe I and Fe II lines. As for the determination

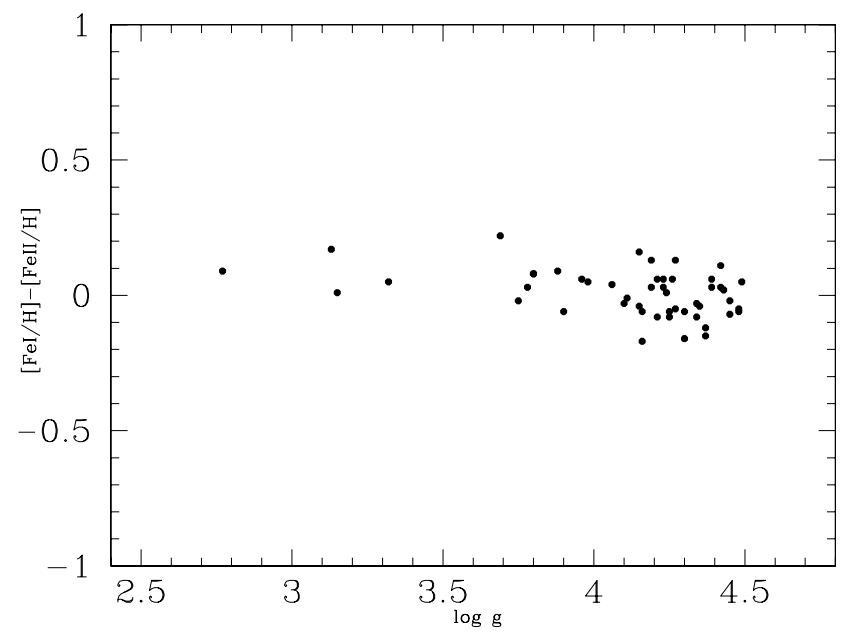

Fig. 5. $[\mathrm{Fe} \mathrm{I} / \mathrm{H}]-[\mathrm{Fe} \mathrm{II} / \mathrm{H}]$ abundance as a function of the stellar surface gravity.

of the Li abundances, we measured the equivalent widths of the $\mathrm{Fe}$ lines (from 3 to 25 lines for Fe I lines and up to 4 lines for Fe II lines) using the code FITLINE. We then derived elemental abundances for the measured line equivalent widths using Turbospectrum (Alvarez \& Plez 1998) taking the latest version of the OSMARCS atmosphere models (e.g., Gustafsson et al. 2008; Plez 2008). Plane parallel 1D models were used for the computations. We adopted the Grevesse \& Sauval (1998) solar abundances.

These lines have been used to compute the Fe abundance but also to check that the ionization equilibrium is consistent with the adopted gravity as shown in Fig. 5. The average $[\mathrm{Fe} / \mathrm{H}]$ from Fe I line is of $-0.54 \pm 0.10$ dex, while the average from Fe II line is of $-0.53 \pm 0.14$ dex. For the three brightest stars, we found a higher Fe abundance than the rest of the sample. This result is in line with the ongoing discussion about the systematic slight differences which can found between $\mathrm{Fe}$ abundances determined in dwarfs and giants.

We also determined the abundance of $\mathrm{Ca}, \mathrm{Ti}$ and $\mathrm{Si}$ in stars where lines were detectable using the linelist of Pace et al. (2010). We finally obtained the abundance of Ca in 76 stars, the abundance of $\mathrm{Ti}$ in 33 stars, and the abundance of $\mathrm{Si}$ in 13 stars.

For calcium, we also measured the equivalent width of four to six lines found in this list to determine the mean $[\alpha / \mathrm{Fe}]$ for the cluster. The mean $[\mathrm{Ca} / \mathrm{Fe}]$ abundance for NGC 2243 based on the determination of $\mathrm{Ca}$ in 76 stars gives $[\mathrm{Ca} / \mathrm{Fe}]=0.00 \pm 0.14$ dex, in agreement within the error bars with the slightly higher value found by Gratton \& Contarini (1994).

The origin of this low value is probably related to the difference in the oscillator strength determination used in Gratton \& Contarini (1994) and Pace et al. (2010). The aim of this work was to determine the Li abundance in a sample of stars around the TO and it was not focused on the determination of the $[\alpha / \mathrm{Fe}]$ ratios, so the instrumental setup was not optimized for it. Because of the limited wavelength range used in this study (however for a large number of stars), we could not find a single Ca line in common with other abundance studies on NGC 2243. Therefore, this solar $[\mathrm{Ca} / \mathrm{Fe}]$ should be considered with caution. We derived a mean Ti abundance of $[\mathrm{Ti} / \mathrm{Fe}]=0.20 \pm 0.22 \mathrm{dex}$ and a Si mean abundance $[\mathrm{Si} / \mathrm{Fe}]=0.12 \pm 0.20 \mathrm{dex}$. In conclusion, our results (i.e., $[\alpha / \mathrm{Fe}]$ abundances ranging from 0.00 to $0.2 \mathrm{dex}$ ) are in fair agreement with the low $[\alpha / \mathrm{Fe}]$ enhancement found in other open clusters or thin disk stars of this metallicity $([\mathrm{Fe} / \mathrm{H}] \simeq-0.5 \mathrm{dex})$. 


\subsection{Error estimates}

The error in the determination of the Li abundances are a combination of the error in the measurement of the equivalent widths and the errors induced by uncertainties of the atmospheric parameters. Errors in the determination of the equivalent widths have been estimated by changing the continuum position. The resulting errors range from 0.05 dex to 0.10 dex. The errors due to the uncertainties in the atmospheric parameters have been computed by evaluating the impact on the $\mathrm{Li}$ abundance of a $T_{\text {eff }}$ variation by $100 \mathrm{~K}, \log g$ by $0.2 \mathrm{dex}$, and $\xi$ by $0.5 \mathrm{~km} \mathrm{~s}^{-1}$ on the adopted atmospheric parameters. These values are based on the uncertainties found in the literature for the color excess and the distance modulus of the cluster. An error of 0.02 on the color index corresponds to an error of $\simeq 100 \mathrm{~K}$ in the temperature scale. An error on the distance modulus of 0.3 corresponds to a change in the surface gravity of 0.2 dex. We added a typical uncertainty of $0.5 \mathrm{~km} \mathrm{~s}^{-1}$ for the microturbulent velocity. As all the measured lines are on the linear part of the curve of growth, the error associated to this parameter is negligible.

The resulting errors have been summed quadratically and give a typical error on the $\mathrm{Li}$ abundance of 0.14 dex. The final errors on lithium are of the order of 0.18 dex. Errors on the Fe abundances, estimated by the line-to-line scatter, give values of 0.1 to 0.2 dex. Summing quadratically the errors coming from uncertainties in atmospheric parameters leads to errors ranging from 0.15 to 0.22 dex.

The abundance uncertainties are dominated by the error in effective temperature. The chosen reddening from Kaluzny et al. (1996) is on the high side of the different estimations and our effective temperatures are in fact higher by about $200 \mathrm{~K}$ compared to the low temperature scale adopted, for instance by Hill \& Pasquini (2000). A difference of this magnitude would shift our abundances by accounting for a lower $\mathrm{Li}$ of about $0.15 \mathrm{dex}$ and a lower $[\mathrm{Fe} / \mathrm{H}]$ of 0.15 dex and a change in the ionization balance $\mathrm{Fe} \mathrm{I}-\mathrm{Fe}$ II by 0.12 dex. Instead the effect on the $[\mathrm{Ca} / \mathrm{Fe}]$ ratio is negligible. Our temperature scale is consistent with that of Kaluzny et al. (1996); this is not surprising given the adoption of the same photometry and reddening.

\section{Discussion}

Figure 6 shows the position of the stars on a $T_{\text {eff }}-V$ space, similar to a color magnitude diagram. It should be remembered that the Hill \& Pasquini (2001) points are on a lower temperature scale than that adopted. As explained above, their points would be $\sim 0.15$ dex higher and $\sim 200 \mathrm{~K}$ moved to the left if they were on the same temperature scale adopted in this work. Figure 7 shows the Li abundances as a function of the $V$ magnitude of the star.

\subsection{The lithium dip}

The Li dip is a feature first observed in the Hyades OC. In a narrow temperature range, the $\mathrm{Li}$ abundance is strongly depleted by factors up to 100 , as first shown by Boesgaard \& Tripicco (1986), and subsequently studied by several authors. Balachandran (1995) compared several clusters in a homogeneous analysis concluding that the mass to which the dip occurs depends on the stellar metallicity, while the ZAMS $T_{\text {eff }}$ does not. With NGC 2243 we have the opportunity to greatly enlarge the metallicity range explored.

For many years the cause of the "extra mixing" producing the Li dip have been debated (Talon \& Charbonnel 2010, and

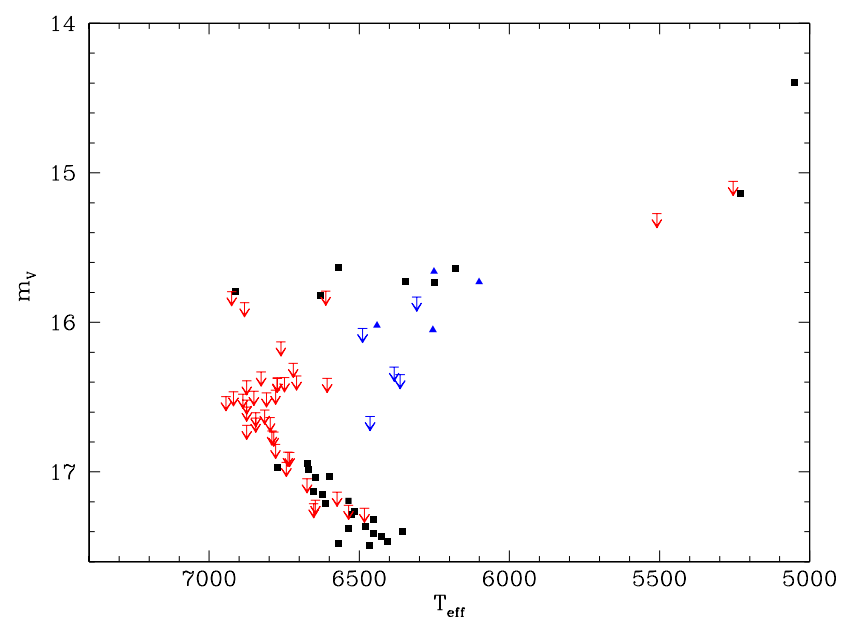

Fig. 6. $V$ magnitude as a function of stellar effective temperature. Black squares are measurements for our targets. Red arrows represent upper limits of our Li abundance measurements. Blue symbols are results from Hill \& Pasquini (2001), where arrows represent upper limits.

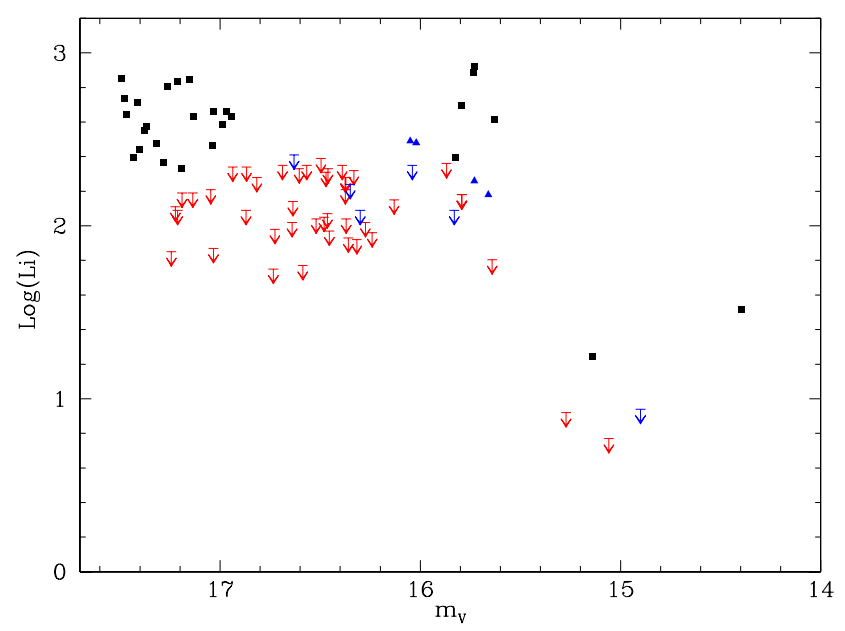

Fig. 7. Lithium abundance as a function of $V$ magnitude. Symbols are the same as in Fig. 6.

references therein). Recently, Smiljanic et al. (2010) succeeded in reproducing the $\mathrm{Li}$ and $\mathrm{Be}$ behavior of the hot side of the Li dip in the IC 4651 cluster by using intermediate mass stars as low as $\sim 1.2 M_{\odot}$ and rotating models (Charbonnel \& Lagarde 2010).

Figure 8 shows the results of the abundance determination in NGC 2243 as a function of the temperature of the star. This figure reveals the clear presence of upper limits in a narrow range of temperature starting around $T_{\text {eff }}=6700 \mathrm{~K}$. The range of temperature $6700-6900 \mathrm{~K}$ represents the Li dip position for this cluster. When informations from Figs. 8 and 6 are put together, one can see that the stars without a Li detection are located in the upper (hottest) part of the ZAMS. Some non detections are also found for cooler (so fainter) stars. We attribute the lack of detection in the faint stars to the resulting low $\mathrm{S} / \mathrm{N}$ ratio in their spectra. Star 1273, with a temperature $T_{\text {eff }}=6913 \mathrm{~K}$ and a magnitude of $V=15.8 \mathrm{mag}$, shows a $\mathrm{Li}$ abundance of 2.70 dex and seems to be the first star located just on the blue side of the dip.

Once proven beyond any doubt the presence of the dip in this metal-poor cluster, we may discuss some additional points. The effective temperature of the dip is higher than that observed 


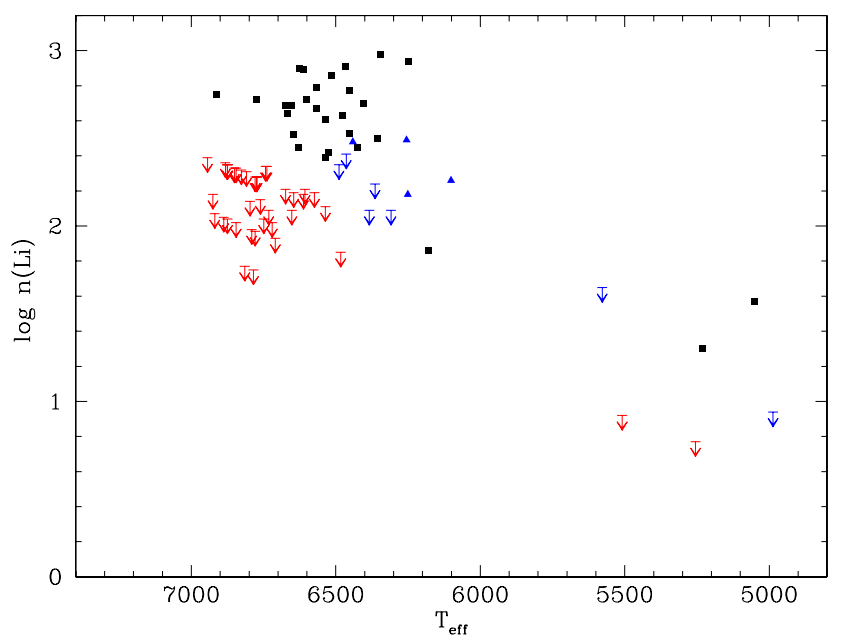

Fig. 8. Abundance of $\mathrm{Li}$ as a function of the effective temperature. Symbols are the same as in Fig. 6.

in other clusters. The mass of the dip can be estimated with the help of evolutionary isochrones. Using the Girardi (web page ${ }^{4}$ ) tracks, a cluster with a turnoff of $6900 \mathrm{~K}$ and a metal content of $[\mathrm{Fe} / \mathrm{H}]=-0.7$ is expected to have an age of $3.5 \mathrm{Gyr}$ and a turnoff mass of $1.12 M_{\odot}$. Using the same isochrone, we could derive approximative masses for the eclipsing binary studied by Kaluzny et al. (2006), finding $0.97 M_{\odot}$ for their components, in fair agreement with the estimated mass by Kaluzny et al. (2006) of 1.089 and $1.069 M_{\odot}$. Positioning the red side of the Li dip at $6700 \mathrm{~K}$, the expected mass of the red edge of the dip would be of $1.03 M_{\odot}$.

The Li dip in this metal-poor cluster is therefore characterized by higher temperatures and substantially lower masses compared to more metal-rich clusters. In the Hyades and Praesepe clusters, the dip center is at about $1.4 M_{\odot}$ (Balachandran 1995). In IC 4651, with $[\mathrm{Fe} / \mathrm{H}]=0.11$, Smiljanic et al. (2010) did not compute the mass of the center, but estimated the red edge of the dip to be at $1.2 M_{\odot}$. In general, the decrease of the Li dip mass with metallicity is very clear, and the effect is clearly visible in NGC 2243, given its low metallicity. Adopting a temperature scale cooler by $\simeq 200 \mathrm{~K}$ degrees would move the dip towards cooler stars, which means also lower masses. Thus the use of a cooler $T_{\text {eff }}$ scale would not change this result.

Figure 9 shows the position of the dip cool-side expressed in mass as a function of the metallicity $[\mathrm{Fe} / \mathrm{H}]$ for a sample of $\mathrm{OCs}$ (Cummings et al. 2012 and references therein). With the addition of NGC 2243 , the $[\mathrm{Fe} / \mathrm{H}]$ range covered has a metallicity of almost 1 dex. This figure reveals a rather tight correlation over the whole range of metallicity. This dependence of the Li dip mass from the metallicity seems to be a well-established feature that models should reproduce.

\subsection{Li chemical evolution}

Observations of many clusters have shown that the regions in the color diagram at the edge of the Li dip show the highest level of $\mathrm{Li}$ abundances, and probably indicate the $\mathrm{Li}$ abundance of the pristine gas. NGC 2243 is not an exception and the five stars bluer to the dip show an average Li abundance of $2.70 \pm 0.20 \mathrm{dex}$. We may assume this value to be a conservative lower limit of the pristine $\mathrm{Li}$ abundance in the cluster. This value

\footnotetext{
4 http://stev.oapd.inaf.it/ lgirardi/
}

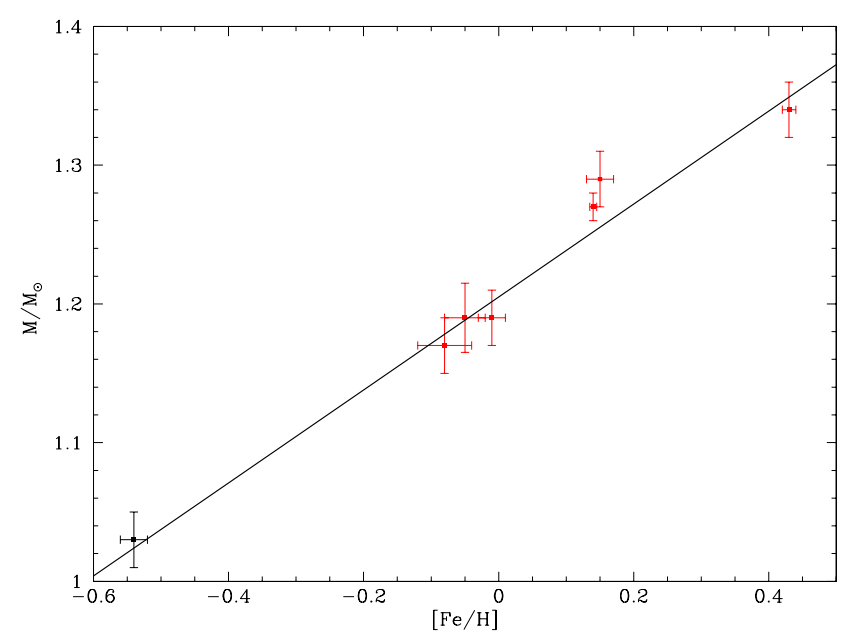

Fig. 9. Correlation of the dip cool-side position in mass and the cluster's $[\mathrm{Fe} / \mathrm{H}]$ ratio. The black symbol represents our result for NGC 2243; red symbols are data from the literature. The black line represents a linear regression fit to the data. The slope value is 0.33 and the correlation coefficient is 0.98 .

is consistent, within errors, with the upper envelope of Li abundances observed in thin disk stars of metallicity similar to that of NGC 2243. Lambert \& Reddy (2004) in their Table 2 provide the mean $\mathrm{Li}$ abundance for the six stars richest in $\mathrm{Li}$ in each metallicity bin and for the bin -0.4 to -0.6 this is $2.64 \pm 0.07 \mathrm{dex}$. The recent large sample of Ramírez et al. (2012) is consistent with this value. The models for Li evolution of Prantzos (2012) predict a value of $\mathrm{Li}$ at this metallicity that is about a factor of two higher. Thus, in order to make these models compatible with the observations, it is necessary to assume that even the stars with the highest observed $\mathrm{Li}$ abundances at this metallicity have depleted Li by a factor of two. This is a direct consequence of the fact that these models assume that the primordial level of $\mathrm{Li}$ is provided by the baryonic density measured from the fluctuations of the Cosmic Microwave Background by the WMAP satellite (Larson et al. 2011; Komatsu et al. 2011), coupled with standard Big Bang nucleosynthesis. The same models, assuming the level of the Spite plateau (Spite \& Spite 1982; Sbordone et al. 2010) as primordial Li abundance, would be in good agreement with the upper envelope of $\mathrm{Li}$ abundances in thin disk stars at all metallicities, without the need of extra Li depletion. For a review on the evolution of Li see also Matteucci (2010). Howk et al. (2012) estimate from the ISM the Li abundance of the Small Magellanic Cloud equal to $2.68 \pm 0.16$ dex for a metallicity of $[\mathrm{Fe} / \mathrm{H}]=-0.59$ dex. Our estimate is $\log n(\mathrm{Li})=2.70 \pm 0.20 \mathrm{dex}$ for $[\mathrm{Fe} / \mathrm{H}]=-0.54 \mathrm{dex}$, therefore in close agreement with the ISM measurements at the same metallicity. Our results would tend to confirm the finding by Howk et al. (2012) that the $\mathrm{Li}$ evolution is extremely constrained when the Li primordial abundance from WMAP is assumed.

\subsection{Comparison with 47 Tuc}

As already mentionned in the introduction, one of the interesting characteristics of NGC 2243 is that its metallicity almost overlaps with that of the well-studied globular cluster 47 Tuc.

The highest Li abundance in NGC 2243 is, as in all young OCs, found at the blue edge of the Li dip and is of $2.70 \pm 0.2 \mathrm{dex}$, with values potentially as high as 2.9 dex. 47 Tuc has been studied in the past by several groups. It was first observed by Pasquini \& Molaro (1997) who found three stars at a level of 
$\log n(\mathrm{Li})=2.35 \mathrm{dex}$, but these observations, made with the $3.5 \mathrm{~m}$ NTT telescope, had a low $\mathrm{S} / \mathrm{N}$ ratio. With the advent of the VLT, higher quality observations and more objects became available. Bonifacio et al. (2007) observed four stars, finding evidence of strong Li variability and a possible correlation with other light elemental abundances, typical of globular clusters. This result was later confirmed by D'Orazi et al. (2010) who have used FLAMES/GIRAFFE data for one hundred stars, showing that a large $\mathrm{Li}$ variation is present. Interestingly, this $\mathrm{Li}$ variation is not related to the $\mathrm{Na}-\mathrm{O}$ correlation found in this cluster.

The extensive work of D'Orazi et al. (2010) confirms the presence of a very large $\mathrm{Li}$ variation in this cluster, with $\mathrm{Li}$ between 2.4 and 2.5 dex and one object as high as 2.78 dex. Moreover, the maximum observed Li abundance in 47 Tuc stars is about 0.2 dex lower than in NGC 2243. It is not easy, however, to firmly establish whether these abundances are representative of the pristine $\mathrm{Li}$ abundance of the cluster gas. While this is the case for NGC 2243 (stars bluer than the gap do not show evidenc, either observational or theoretical, for Li depletion), this is not the case for 47 Tuc. The turnoff stars of this cluster are rather cool ( $T_{\text {eff }}$ less than $\simeq 5800 \mathrm{~K}$ ), more than 500 degrees $\mathrm{K}$ cooler than the faintest stars observed in NGC 2243. At this effective temperature, old OC stars already show evidence of main sequence Li depletion (Pasquini 2000; Sestito \& Randich 2005). It is important to note that none of the OCs studied so far is as metal-poor as 47 Tuc or NGC 2243; it would be very important to observe fainter stars in NGC 2243 to confirm the Li trend with effective temperature. Still, the possibility that the Li observed in 47 Tuc stars does not correspond to the pristine $\mathrm{Li}$, but to slightly depleted Li, cannot be excluded. Keeping this possibility in mind, it is worth analyzing the case that the $\mathrm{Li}$ abundance in the two clusters was not initially the same.

Given its old age, in spite of its high metallicity, it would be natural to assume that 47 Tuc started from the primordial $\mathrm{Li}$ abundance, $\log n\left({ }^{7} \mathrm{Li}\right)=2.72 \mathrm{dex}$, from WMAP+SBBN (Cyburt et al. 2008). Instead, NGC 2243, with a much younger age, should have incorporated the Li produced by the Galactic sources, thus $\log n\left({ }^{7} \mathrm{Li}\right) \sim 3.0 \mathrm{dex}$, according to the models of Prantzos (2012) at this metallicity. If this is the case, one has to conclude that the Li depletion with time is a highly non-linear process. In fact, the two clusters started with a difference in $\mathrm{Li}$ of a factor of 2 and they retain this difference, in spite of the fact that in 47 Tuc the Li has had 8 Gyr more to accomplish its depletion. Another intriguing aspect of this scenario is that the amount of depletion (initial/present) is again roughly a factor of two in both cases. The conclusion could be that $\mathrm{Li}$ is depleted very rapidly, by a factor of two, from whatever value it starts from, and then remains constant for all of the star's lifetime without taking into account the "extra mixing" that enters into play to explain the "Li dip" and the cooler stars. This scenario could be consistent with the suggestion that the Li depletion takes place during the pre-main sequence (Molaro et al. 2012). However, the observational evidences show that pre-main Sequence (PMS) depletion in solar stars is not really effective (Randich et al. 2001; Jeffries 2006). Considering the relatively low metal abundance of these clusters compared to the Sun, even less PMS depletion would be theoretically expected.

This promising picture is, however, troubled if we note that the star with the highest level of Li abundance in the D'Orazi et al. (2010) sample has $\log n\left({ }^{7} \mathrm{Li}\right)=2.78$, i.e., close to the highest value also observed in NGC 2243. This "upper limit" of the Li content in 47 Tuc stars can be considered the closest value to the original Li content of the cluster.
This value would then suggest that the two clusters started with similar Li abundance. The implications of this are puzzling. We see three possible situations:

1. Li is depleted both in 47 Tuc and NGC 2243, 47 Tuc already benefitted from an initial $\mathrm{Li}$ that was a factor of two higher than the primordial value and thereafter the Li abundance was constant up to the time of the NGC 2243 cluster formation;

2. neither 47 Tuc nor NGC 2243 have depleted any Li and were formed with the primordial abundance. Again no Li is produced between 12 Gyr ago and 4 Gyr ago;

3. 47 Tuc started with the primordial $\mathrm{Li}$ abundance, but its highest Li stars suffered no Li depletion. NGC 2243 started with a higher Li-abundance and its highest Li stars suffered depletion by a factor of two.

All of these contradict our current understanding of Li evolution and stellar structure. It would certainly be useful to extend the work of D'Orazi et al. (2010) and have new determinations of the $\mathrm{Li}$ abundance in $47 \mathrm{Tuc}$, to check if this very high Li abundance found in one star is real and is not simply a spurious result due to a low $\mathrm{S} / \mathrm{N}$ spectrum or incorrect stellar parameters.

If the Li initial abundance were the same between the two clusters, then the Li enrichment would basically be determined by the Fe content rather than by the star formation history of the two clusters, which was quite different, as shown by their different $[\alpha / \mathrm{Fe}]$ enrichment.

\section{Conclusion}

VLT-FLAMES observations of a sample of stars belonging to NGC 2243 has permitted us to determine the Li abundances in 27 stars and the $\mathrm{Fe}$ and $\mathrm{Ca}$ abundances in 76 stars. We found a metallicity of $[\mathrm{Fe} / \mathrm{H}]=-0.54 \pm 0.10 \mathrm{dex}$ and $[\mathrm{Ca} / \mathrm{Fe}]=$ $0.00 \pm 0.14 \mathrm{dex}$. The $\mathrm{Li}$ dip is well defined with a center corresponding to a mass of $M=1.06 M_{\odot}$, a value much smaller than the value found for solar metallicity OCs. We confirm a correlation between the Li dip cool-side position in mass as a function of the cluster's $[\mathrm{Fe} / \mathrm{H}]$ ratio, extending the relation towards metallicity down to $[\mathrm{Fe} / \mathrm{H}] \simeq-0.55$ dex. A Li abundance of $2.70 \pm 0.2$ dex found at the blue edge of the Li dip of NGC 2243 is in good agreement with results obtained for the $\mathrm{Li}$ of the ISM in the Small Magellanic Cloud having similar low metallicity. This value of $\mathrm{Li}$ in metal-poor objects is comparable to the Li abundance deduced from WMAP measurements putting strong constraints on the models of Li enrichment during the early history of our Galaxy.

Acknowledgements. The authors are grateful to the anonymous referee for a very careful reading of the paper and valuable comments. P.F. acknowledges support from the ESO Visitor program. K.B. was supported by the ESO DGDF 2008, and by the Italian Ministero dell'Istruzione, Università e Ricerca (MIUR) fundings. We thank Simone Zaggia for the discussions about the GIRAFFE code girBLDRS. P.F. and P.B. acknowledge support from the Programme National de Physique Stellaire (PNPS) and the Programme National de Cosmologie et Galaxies (PNCG) of the Institut National de Sciences de 1'Universe of CNRS. This research made use of WEBDA, operated at the Institute for Astronomy of the University of Vienna, and of SIMBAD/VIZIER databases operated at the CDS (Strasbourg, France)

\section{References}

Alonso, A., Arribas, S., \& Martínez-Roger, C. 1999, A\&AS, 140, 261

Alvarez, R., \& Plez, B. 1998, A\&A, 330, 1109

Balachandran, S. 1995, ApJ, 446, 203

Bergbusch, P. A., Vandenberg, D. A., \& Infante, L. 1991, AJ, 101, 2102 
Blecha, A., Cayatte, V., North P., et al. 2000, SPIE Conf. 4008, eds. M. Iye, \& A. F. Moorwood, 367

Boesgaard, A. M., \& Tripicco, M. J. 1986, ApJ, 302, L49

Bonifacio, P., Pasquini, L., Molaro, P., et al. 2007, A\&A, 470, 153

Bonifazi, A., Tosi, M., Fusi Pecci, F., \& Romeo, G. 1990, MNRAS, 245, 15

Casagrande, L., Ramírez, I., Melèndez, J., Bessel, M., \& Asplund, M. 2010, A\&A, 512, 54

Cayrel, R. 1988, IAU Symp., 132, 345

Charbonnel, C., \& Lagarde, N. 2010, A\&A, 522, A10

Cummings, J., Delyannis, C., Anthony-Twarog, B., Twarog, B., \& Maderak, R. 2012, AJ, 144, 37

Cyburt, R. H., Fields, B. D., \& Olive, K. A. 2008, JCAP, 11, 12

D'Orazi, V., Lucatello, S., Gratton, R., et al. 2010, ApJ, 713, L1

Edvardsson, B., Andersen, J., Gustaffson, B., et al. 1993, A\&A, 275, 101

Gratton, R., \& Contarini, G. 1994, A\&A, 283, 911

Grevesse, N., \& Sauval, A. J. 1998, Space Sci. Rev., 85, 161

Gustafsson, B., Edvardsson, B., Eriksson, K., et al. 2008, A\&A, 486, 951

Hill, V., \& Pasquini, L. 2000, IAU Symp., 198, 293

Howk, J. C., Lehner, N., Fields, B. D., \& Mathews, G. J. 2012, Nature, 489, 121

Jeffries, R. 2006, in Chemical Abundances and Mixing in Stars in the Milky Way and its Satellites, ESO Astrophys. Symp. (Springer-Verlag), 163

Kaluzny, J., Krzemiński, W., \& Mazur, B. 1996, A\&A, 118, 303

Kaluzny, J., Pych, W., Rucinski, S. M., \& Thompson, I. B. 2006, Acta. Astron., 56,237

Koch, A., \& McWilliam, A. 2008, AJ135, 1551
Komatsu, E., Smith, K. M., Dunkley, J., et al. 2011, ApJS, 192, 18 Lambert, D. L., \& Reddy, B. E. 2004, MNRAS, 349, 757

Larson, D., Dunkley, J., Hinshaw, G., et al. 2011, ApJS, 192, 16

Lemasle, B., François, P., Bono, G., et al. 2007, A\&A, 467, 283

Matteucci, F. 2010, IAU Symp., 268, 453

Molaro, P., Bressan, A., Barbieri, M., Marigo, P., \& Zaggia, S. 2012, Mem. Soc. Astron. It. Supp., 22, 233

Pace, G., Danziger, J., Carraro, G., et al. 2010, A\&A, 515, 28

Pasquini, L. 2000, IAU Symp, 198, 269

Pasquini, L., \& Molaro, P. 1997, A\&A, 322, 109

Pasquini, L., Avila, G., Blecha, A., et al. 2002, The Messenger, 110, 1

Plez, B. 2008, Phys. Scr. T, 133, 014003

Prantzos, N. 2012, A\&A, 542, A67

Ramírez, I., Fish, J. R., Lambert, D. L., \& Allende Prieto, C. 2012, ApJ, 756, 46

Randich, S., Pasquini, L., \& Pallavicini, R. 2000, A\&A, 356, L25

Randich, S., Pallavicini, R., Meola, G., Stauffer, J. R., \& Balachandran, S. C. 2001, A\&A, 372, 862

Randich, S., Sestito, P., \& Pallavicini, R. 2003, A\&A, 399, 133

Sbordone, L., Bonifacio P., Caffau, E., et al. 2010, A\&A, 522, 26

Sestito, P., \& Randish, S. 2005, A\&A, 442, 615

Smiljanic, R., Pasquini, L., Charbonnel, C., \& Lagarde, N. 2010, A\&A, 510, 50 Spite, F., \& Spite, M. 1982, A\&A, 115, 357

Talon, S., \& Charbonnel, C. 2010, Proc. IAU, IAU Symp., 268, 365

VandenBerg, D. A., Bergbusch, P. A., \& Dowler, P. D. 2006, ApJS, 162, 375

Venn, K., Irwin, M., Shetrone, M. D., et al. 2004, AJ, 128, 1177 
P. François et al.: Lithium in NGC 2243

Table 2. Stars with well-measured lithium abundance.

\begin{tabular}{|c|c|c|c|c|c|c|c|c|c|c|}
\hline Object & $\begin{array}{c}V \\
(\mathrm{mag})\end{array}$ & $V-I$ & $\begin{array}{l}T_{\text {eff }} \\
(\mathrm{K})\end{array}$ & $\log g$ & {$[\mathrm{Fe} \mathrm{I} / \mathrm{H}]$} & {$[\mathrm{Fe} \mathrm{II} / \mathrm{H}]$} & $\log n(\mathrm{Li})$ & $\begin{array}{c}V_{\mathrm{rad}}^{1} \\
\left(\mathrm{~km} \mathrm{~s}^{-1}\right)\end{array}$ & $\begin{array}{c}V_{\mathrm{rad}}^{2} \\
\left(\mathrm{~km} \mathrm{~s}^{-1}\right)\end{array}$ & $\begin{array}{c}\left\langle V_{\mathrm{rad}}\right\rangle \\
\left(\mathrm{km} \mathrm{s}^{-1}\right)\end{array}$ \\
\hline 148 & 17.037 & 0.504 & 6646 & 4.39 & -0.56 & -0.62 & 2.46 & $60.8 \pm 1.4$ & $60.9 \pm 1.2$ & $60.8 \pm 0.9$ \\
\hline 231 & 17.400 & 0.559 & 6355 & 4.45 & -0.64 & -0.62 & 2.44 & $64.3 \pm 1.3$ & $64.4 \pm 1.5$ & $64.3 \pm 1.0$ \\
\hline 244 & 17.467 & 0.549 & 6405 & 4.49 & -0.61 & & 2.64 & $62.5 \pm 1.7$ & $63.1 \pm 1.5$ & $62.8 \pm 1.1$ \\
\hline 403 & 14.394 & 0.935 & 5051 & 2.77 & -0.33 & -0.42 & 1.51 & $62.7 \pm 0.8$ & $62.6 \pm 0.5$ & $62.7 \pm 0.4$ \\
\hline 414 & 17.412 & 0.540 & 6452 & 4.48 & -0.59 & -0.54 & 2.71 & $63.4 \pm 1.6$ & $62.7 \pm 1.2$ & $63.0 \pm 1.0$ \\
\hline 655 & 16.969 & 0.482 & 6773 & 4.39 & -0.56 & -0.59 & 2.66 & $61.9 \pm 1.9$ & $62.3 \pm 1.1$ & $62.1 \pm 1.1$ \\
\hline 728 & 16.987 & 0.500 & 6668 & 4.37 & -0.55 & -0.40 & 2.58 & $61.8 \pm 1.0$ & $62.3 \pm 1.3$ & $62.0 \pm 0.8$ \\
\hline 766 & 17.315 & 0.540 & 6452 & 4.44 & -0.55 & & 2.47 & $61.1 \pm 1.7$ & $60.8 \pm 1.4$ & $60.9 \pm 1.1$ \\
\hline 873 & 17.377 & 0.524 & 6536 & 4.49 & -0.58 & -0.63 & 2.55 & $60.2 \pm 2.5$ & $60.5 \pm 1.6$ & $60.3 \pm 1.5$ \\
\hline 1106 & 17.477 & 0.518 & 6568 & 4.54 & -0.50 & & 2.73 & $63.5 \pm 1.9$ & $63.1 \pm 1.5$ & $63.3 \pm 1.2$ \\
\hline 1161 & 17.194 & 0.524 & 6536 & 4.42 & -0.76 & -0.87 & 2.33 & $61.6 \pm 1.6$ & $62.0 \pm 1.5$ & $61.8 \pm 1.1$ \\
\hline 1183 & 17.213 & 0.510 & 6612 & 4.45 & -0.43 & & 2.83 & $62.5 \pm 3.0$ & $61.0 \pm 2.3$ & $61.8 \pm 1.9$ \\
\hline 1189 & 17.366 & 0.535 & 6478 & 4.47 & -0.56 & & 2.57 & $61.5 \pm 1.5$ & $61.6 \pm 1.5$ & $61.6 \pm 1.1$ \\
\hline 1241 & 15.824 & 0.507 & 6629 & 3.90 & -0.58 & -0.52 & 2.39 & $60.9 \pm 0.8$ & $60.8 \pm 0.5$ & $60.8 \pm 0.5$ \\
\hline 1273 & 15.796 & 0.459 & 6913 & 3.96 & -0.52 & -0.58 & 2.69 & $61.2 \pm 1.0$ & $61.5 \pm 0.6$ & $61.4 \pm 0.6$ \\
\hline 1284 & 17.284 & 0.526 & 6525 & 4.45 & -0.66 & & 2.36 & $61.1 \pm 2.6$ & $61.9 \pm 2.1$ & $61.5 \pm 1.7$ \\
\hline 1317 & 17.153 & 0.508 & 6624 & 4.43 & -0.57 & & 2.84 & $62.1 \pm 2.0$ & $63.4 \pm 1.5$ & $62.7 \pm 1.3$ \\
\hline 1323 & 17.134 & 0.503 & 6652 & 4.43 & -0.61 & -0.63 & 2.63 & $62.9 \pm 1.0$ & $62.7 \pm 1.2$ & $62.8 \pm 0.8$ \\
\hline 1405 & 17.263 & 0.528 & 6515 & 4.44 & -0.73 & & 2.80 & $62.7 \pm 1.2$ & $61.4 \pm 3.5$ & $62.1 \pm 1.8$ \\
\hline 1469 & 17.434 & 0.545 & 6426 & 4.48 & -0.42 & -0.36 & 2.39 & $63.4 \pm 1.9$ & $62.4 \pm 1.4$ & $62.9 \pm 1.2$ \\
\hline 1506 & 16.944 & 0.499 & 6674 & 4.36 & -0.50 & & 2.63 & $63.5 \pm 1.5$ & $64.3 \pm 1.8$ & $63.9 \pm 1.2$ \\
\hline 1634 & 15.734 & 0.581 & 6249 & 3.75 & -0.49 & -0.47 & 2.88 & $62.7 \pm 0.4$ & $62.0 \pm 0.5$ & $62.4 \pm 0.3$ \\
\hline 1663 & 15.729 & 0.561 & 6345 & 3.78 & -0.48 & -0.51 & 2.92 & $61.1 \pm 2.6$ & $62.1 \pm 1.8$ & $61.6 \pm 1.6$ \\
\hline 1776 & 17.031 & 0.512 & 6601 & 4.37 & -0.74 & -0.62 & 2.66 & $62.0 \pm 0.7$ & $62.0 \pm 0.4$ & $62.0 \pm 0.4$ \\
\hline 1792 & 17.491 & 0.537 & 6467 & 4.52 & -0.66 & & 2.85 & $62.9 \pm 3.2$ & $62.9 \pm 2.0$ & $62.9 \pm 1.9$ \\
\hline 1801 & 15.632 & 0.518 & 6568 & 3.80 & -0.49 & -0.57 & 2.61 & $61.4 \pm 1.3$ & $61.1 \pm 1.6$ & $61.2 \pm 1.0$ \\
\hline 1992 & 15.141 & 0.864 & 5231 & 3.15 & -0.29 & -0.30 & 1.24 & $63.1 \pm 0.8$ & $63.1 \pm 0.5$ & $63.1 \pm 0.5$ \\
\hline
\end{tabular}


Table 3. Stars with upper limits in lithium abundances.

\begin{tabular}{|c|c|c|c|c|c|c|c|c|c|c|}
\hline Object & $\begin{array}{c}V \\
(\mathrm{mag})\end{array}$ & $V-I$ & $\begin{array}{l}T_{\text {eff }} \\
(\mathrm{K})\end{array}$ & $\log g$ & {$[\mathrm{Fe} \mathrm{I} / \mathrm{H}]$} & {$[\mathrm{Fe} \mathrm{II} / \mathrm{H}]$} & $\log n(\mathrm{Li})$ & $\begin{array}{c}V_{\mathrm{rad}}^{1} \\
\left(\mathrm{~km} \mathrm{~s}^{-1}\right)\end{array}$ & $\begin{array}{c}V_{\mathrm{rad}}^{2} \\
\left(\mathrm{~km} \mathrm{~s}^{-1}\right)\end{array}$ & $\begin{array}{c}\left\langle V_{\mathrm{rad}}\right\rangle \\
\left(\mathrm{km} \mathrm{s}^{-1}\right)\end{array}$ \\
\hline 33 & 17.223 & 0.524 & 6536 & 4.43 & -0.58 & & 2.05 & $61.5 \pm 1.5$ & $61.1 \pm 1.8$ & $61.3 \pm 1.2$ \\
\hline 80 & 16.587 & 0.475 & 6815 & 4.25 & -0.54 & -0.48 & 1.71 & $62.6 \pm 0.9$ & $62.7 \pm 1.2$ & $62.6 \pm 0.8$ \\
\hline 120 & 15.791 & 0.510 & 6612 & 3.88 & -0.50 & -0.59 & 2.12 & $64.9 \pm 0.7$ & $64.6 \pm 0.5$ & $64.8 \pm 0.4$ \\
\hline 213 & 16.371 & 0.482 & 6773 & 4.16 & -0.75 & -0.58 & 2.22 & $62.0 \pm 1.2$ & $62.5 \pm 1.3$ & $62.3 \pm 0.9$ \\
\hline 274 & 17.243 & 0.534 & 6483 & 4.42 & -0.58 & -0.61 & 1.79 & $63.9 \pm 1.9$ & $62.7 \pm 1.4$ & $63.3 \pm 1.2$ \\
\hline 354 & 16.636 & 0.478 & 6797 & 4.27 & -0.57 & -0.70 & 2.08 & $62.8 \pm 1.0$ & $62.0 \pm 1.2$ & $62.4 \pm 0.8$ \\
\hline 397 & 17.136 & 0.517 & 6574 & 4.41 & -0.66 & & 2.13 & $63.5 \pm 2.7$ & $63.5 \pm 2.1$ & $63.5 \pm 1.7$ \\
\hline 439 & 16.937 & 0.487 & 6743 & 4.37 & -0.47 & & 2.28 & $62.3 \pm 1.5$ & $63.6 \pm 1.2$ & $62.9 \pm 1.0$ \\
\hline 456 & 16.640 & 0.470 & 6845 & 4.28 & -0.62 & & 1.96 & $61.9 \pm 1.0$ & $62.9 \pm 1.4$ & $62.4 \pm 0.9$ \\
\hline 485 & 16.870 & 0.489 & 6732 & 4.34 & -0.63 & -0.60 & 2.03 & $61.9 \pm 1.0$ & $61.7 \pm 1.3$ & $61.8 \pm 0.8$ \\
\hline 520 & 16.688 & 0.465 & 6875 & 4.31 & -0.66 & & 2.29 & $62.7 \pm 1.9$ & $61.8 \pm 2.3$ & $62.2 \pm 1.5$ \\
\hline 523 & 16.274 & 0.491 & 6720 & 4.10 & -0.56 & -0.53 & 1.96 & $64.0 \pm 1.0$ & $64.5 \pm 0.8$ & $64.2 \pm 0.7$ \\
\hline 529 & 16.131 & 0.484 & 6761 & 4.06 & -0.47 & -0.51 & 2.09 & $62.8 \pm 1.0$ & $63.2 \pm 0.8$ & $63.0 \pm 0.6$ \\
\hline 547 & 16.240 & 0.486 & 6749 & 4.10 & -0.36 & -0.36 & 1.90 & $62.5 \pm 0.8$ & $62.5 \pm 0.6$ & $62.5 \pm 0.5$ \\
\hline 577 & 15.641 & 0.596 & 6179 & 3.69 & -0.39 & -0.61 & 1.74 & $61.7 \pm 0.4$ & $61.9 \pm 0.5$ & $61.8 \pm 0.3$ \\
\hline 612 & 16.332 & 0.473 & 6827 & 4.15 & -0.44 & -0.60 & 2.26 & $62.2 \pm 1.2$ & $62.2 \pm 1.3$ & $62.2 \pm 0.9$ \\
\hline 631 & 15.272 & 0.770 & 5509 & 3.32 & -0.51 & -0.56 & 0.86 & $64.1 \pm 0.6$ & $64.2 \pm 0.4$ & $64.1 \pm 0.3$ \\
\hline 657 & 16.374 & 0.511 & 6607 & 4.11 & -0.68 & -0.67 & 2.15 & $61.0 \pm 1.0$ & $61.3 \pm 1.0$ & $61.1 \pm 0.7$ \\
\hline 684 & 17.033 & 0.507 & 6629 & 4.38 & -0.69 & & 1.81 & $61.1 \pm 2.1$ & $61.6 \pm 1.7$ & $61.3 \pm 1.4$ \\
\hline 700 & 17.212 & 0.503 & 6652 & 4.46 & -0.52 & & 2.03 & $61.6 \pm 2.2$ & $62.0 \pm 1.7$ & $61.8 \pm 1.4$ \\
\hline 770 & 16.734 & 0.480 & 6785 & 4.30 & -0.61 & -0.45 & 1.69 & $62.6 \pm 1.1$ & $62.4 \pm 1.2$ & $62.5 \pm 0.8$ \\
\hline 784 & 16.726 & 0.479 & 6791 & 4.30 & -0.56 & -0.50 & 1.92 & $61.1 \pm 1.3$ & $60.8 \pm 1.3$ & $61.0 \pm 0.9$ \\
\hline 838 & 15.869 & 0.464 & 6882 & 3.98 & -0.54 & -0.59 & 2.30 & $61.2 \pm 0.8$ & $61.7 \pm 0.7$ & $61.5 \pm 0.5$ \\
\hline 863 & 15.794 & 0.457 & 6925 & 3.96 & -0.49 & & 2.12 & $61.9 \pm 1.1$ & $62.2 \pm 1.0$ & $62.0 \pm 0.8$ \\
\hline 875 & 17.190 & 0.504 & 6646 & 4.45 & -0.65 & -0.58 & 2.13 & $62.7 \pm 2.0$ & $62.4 \pm 1.4$ & $62.6 \pm 1.2$ \\
\hline 1035 & 16.464 & 0.458 & 6919 & 4.23 & -0.51 & -0.54 & 2.01 & $61.8 \pm 0.8$ & $62.1 \pm 0.8$ & $61.9 \pm 0.6$ \\
\hline 1100 & 16.390 & 0.465 & 6875 & 4.19 & -0.60 & -0.73 & 2.29 & $62.9 \pm 1.3$ & $62.3 \pm 1.3$ & $62.6 \pm 0.9$ \\
\hline 1253 & 16.370 & 0.486 & 6749 & 4.15 & -0.68 & -0.64 & 1.98 & $61.4 \pm 1.4$ & $61.8 \pm 0.9$ & $61.6 \pm 0.8$ \\
\hline 1299 & 16.868 & 0.488 & 6738 & 4.35 & -0.52 & -0.48 & 2.28 & $62.1 \pm 1.2$ & $62.1 \pm 1.1$ & $62.1 \pm 0.8$ \\
\hline 1321 & 16.605 & 0.470 & 6845 & 4.27 & -0.45 & -0.40 & 2.27 & $62.4 \pm 1.2$ & $61.8 \pm 1.0$ & $62.1 \pm 0.8$ \\
\hline 1339 & 16.454 & 0.481 & 6779 & 4.19 & -0.59 & -0.62 & 1.91 & $65.3 \pm 1.5$ & $65.4 \pm 1.2$ & $65.4 \pm 1.0$ \\
\hline 1375 & 16.471 & 0.476 & 6809 & 4.21 & -0.59 & -0.51 & 2.25 & $62.0 \pm 2.0$ & $62.4 \pm 1.4$ & $62.2 \pm 1.2$ \\
\hline 1566 & 16.375 & 0.482 & 6773 & 4.16 & -0.44 & -0.38 & 2.22 & $61.7 \pm 2.3$ & $61.9 \pm 1.1$ & $61.8 \pm 1.3$ \\
\hline 1590 & 16.317 & 0.495 & 6697 & 4.11 & -0.54 & -0.50 & 1.86 & $62.8 \pm 0.8$ & $63.0 \pm 0.8$ & $62.9 \pm 0.6$ \\
\hline 1597 & 16.567 & 0.465 & 6875 & 4.26 & -0.51 & -0.57 & 2.29 & $61.8 \pm 1.6$ & $62.5 \pm 1.0$ & $62.1 \pm 0.9$ \\
\hline 1635 & 17.046 & 0.499 & 6674 & 4.40 & -0.51 & & 2.15 & $61.6 \pm 1.9$ & $60.7 \pm 1.5$ & $61.1 \pm 1.2$ \\
\hline 1648 & 15.058 & 0.855 & 5255 & 3.13 & -0.16 & -0.33 & 0.71 & $62.0 \pm 0.3$ & $62.1 \pm 0.2$ & $62.1 \pm 0.2$ \\
\hline 1687 & 16.520 & 0.465 & 6875 & 4.24 & -0.47 & -0.48 & 1.98 & $62.9 \pm 1.3$ & $62.0 \pm 0.9$ & $62.4 \pm 0.8$ \\
\hline 1709 & 16.481 & 0.463 & 6888 & 4.23 & -0.47 & -0.53 & 1.99 & $61.9 \pm 1.4$ & $61.8 \pm 1.1$ & $61.9 \pm 0.9$ \\
\hline 1751 & 16.461 & 0.469 & 6851 & 4.21 & -0.42 & -0.48 & 2.27 & $63.1 \pm 1.2$ & $62.7 \pm 0.9$ & $62.9 \pm 0.7$ \\
\hline 1752 & 16.359 & 0.493 & 6709 & 4.13 & -0.62 & & 1.87 & $62.0 \pm 1.7$ & $61.6 \pm 1.1$ & $61.8 \pm 1.0$ \\
\hline 1839 & 16.816 & 0.481 & 6779 & 4.34 & -0.62 & -0.54 & 2.22 & $62.1 \pm 1.3$ & $61.0 \pm 1.5$ & $61.5 \pm 1.0$ \\
\hline 1874 & 16.496 & 0.454 & 6944 & 4.25 & -0.52 & -0.44 & 2.33 & $63.6 \pm 1.6$ & $62.1 \pm 1.2$ & $62.8 \pm 1.0$ \\
\hline
\end{tabular}

